\title{
Flexion Osteotomy in Genu Recurvatum Following Post-Polio Syndrome: Use of an Old Technique in a New Condition
}

\author{
Abolfazle Bagherifard, ${ }^{1}$ Mohamad Rahbar, ${ }^{1}$ Ali Shahsavari, ${ }^{1, *}$ Mehdi Moaiedfar,, and Mehrdad \\ Bahrabadi $^{1}$ \\ ${ }^{1}$ Bone and Joint Reconstruction Research Center, Shafa Orthopedic Hospital, Iran University of Medical Sciences, Tehran, IR Iran \\ "Corresponding author: Ali Shahsavari, Bone and Joint Reconstruction Research Center, Shafa Orthopedic Hospital, Iran University of Medical Sciences, Tehran, Iran. Tel: \\ +98-2133542000-8, Fax: +98-2133542020, E-mail: ali.shahsavari2@gmail.com
}

Received 2017 January 16; Revised 2017 February 28; Accepted 2017 April 04.

\begin{abstract}
Introduction: Post-polio syndrome(PPS) can have devastating functional effects on the walking ability of patients decades after the acute disease. Genu recurvatum, as a consequence of PPS, is one such disability which can be treated through different measures. Case Presentation: A 43-year-old woman with a history of supracondylar extension osteotomy of the left femur at the age of 22 was admitted to our hospital for a flexion contracture of the left knee due to poliomyelitis. She was able to walk without assistance for 20 years after the osteotomy until one year ago, when she started to experience progressive genu recurvatum. In the clinical and laboratory workup, she was diagnosed with PPS. Accordingly, we decided to perform supracondylar flexion osteotomy.

Conclusions: Supracondylar flexion osteotomy in patients with genu recurvatum, as a consequence of PPS, is a valuable treatment, which can relieve the patients' dependence on walking aids and improve their symptoms.
\end{abstract}

Keywords: Poliomyelitis, Osteotomy, Post-Polio Syndrome, Genu Recurvatum

\section{Introduction}

Post-polio syndrome (PPS) is the term used for the late manifestations of polio, occurring 30 to 40 years after the acute disease (1). Although PPS has been recognized for more than a century, it is more common today due to the epidemics of polio in previous decades (2). The manifestations of PPS include general, neurological, and musculoskeletal disorders. Fatigue is the most common general manifestation of PPS $(3,4)$.

Slowly progressive muscle weakness is the most important neurological manifestation, which is reported in most patients, and is more likely to occur in the muscle groups previously affected by poliomyelitis (5). Pain caused by joint instability is the main musculoskeletal problem in PPS (1). Weakness leads to the overstretching of the joints and pain (1), thereby increasing the risk of new deformities.

Genu recurvatum, when mild, confers knee stability due to the self-locking mechanism of the knee. With further hyperextension, the knee collapses posteriorly, as the center of gravity of the body passes anterior to the knee joint. This leads to increasing energy expenditure during walking due to increased vertical movements of the center of gravity of the body (6), as well as progressive genu recurvatum caused by chronic stretching of the posterior soft tissues of the knee (often painful) (7).

\section{Case Presentation}

The patient was a 43-year-old woman with a history of poliomyelitis at the age of 3. The diagnosis of poliomyelitis was established by careful review of the patient's medical records. Due to the endemic course of poliomyelitis in Iran in previous decades, diagnosis in our case required great accuracy. The patient had suffered from progressive flexion contracture of the knee, resulting in limping during childhood; accordingly, she had undergone supracondylar extension osteotomy at the age of 22 . She experienced functional recovery and could walk without a brace or crutches for 20 years after the surgery.

At the age of 42 , the patient experienced progressive genu recurvatum, leading to her disability and loss of walking potential. She did not report any history of trauma and diabetes, collagen vascular disease, and polyneuropathy were ruled out through clinical and laboratory workup. In addition, there was no evidence of compression neuropathy due to the use of crutches. Neurological examination was performed by 2 neurologists in our institution. In addition, motor strength was assessed based on the scale developed by the medical research council (8). PPS was diagnosed, based on the criteria proposed by Jubelt and updated by the post-polio task force (1).

For the correction of genu recurvatum, we decided to perform supracondylar flexion osteotomy; this decision 
was made by a team of knee surgeons in our institute. After the induction of anesthesia, the passive range of motions in the knee was assessed $\left(50^{\circ}-0^{\circ}-140^{\circ}\right)$. During the operation, the genu recurvatum was corrected from $50^{\circ}$ to $5^{\circ}$ of hyperextension through opening up the osteotomy site. After this correction, we used a bone block allograft and a locking plate to fill and fix the osteotomy site, respectively.

After surgery, the patient used a knee immobilizer for 3 weeks and was non-weight-bearing for 3 months. In the final follow-up, she was able to walk without crutches or brace. The present study was approved by the ethics board of our center, and a written consent was obtained from the patient to publish the results of surgery.

\section{Discussion}

PPS may be a result of recovery from a polio attack. After the attack, nerve fibers sprout to innervate more muscle fibers than normal. These hyperfunctional motor neurons cannot meet the metabolic requirements of the sprouts, and consequently, slow deterioration of the terminals may gradually occur $(9,10)$. Also, weakness appears after an adequate number of nerve terminals are destroyed.

Genu recurvatum is a manifestation of PPS (7) and can be managed by conservative or surgical measures. The nonsurgical method of treatment is based on the use of a hinged leg orthosis, which can prevent the progression of genu recurvatum. This type of orthosis has many disadvantages, including its bulky design and feeling of discomfort when seated. It is also difficult to wear by patients with muscular weakness $(11,12)$.

Arthrodesis is a surgical method for the treatment of genu recurvatum. Also, anterior bone block technique is a method of treatment in paralytic genu recurvatum (13-15), which was first described by Campbell in 1918 (16). The graft has the same relationship with the tibia as the olecranon process with the ulna; the surgery is literally a conversion of the knee joint into an elbow (15). In this technique, the articular cartilage and anterior cortex are removed from the proximal half of the patella.

A transverse trough is made in the tibial plateau between the menisci, and the patella is placed within so that the articular surface of the patella faces the articular surface of the femoral condyles. The position of the patella is adjusted in a way that $5^{\circ}$ hyperextension is allowed at the end of the procedure. The patella is fixed to the tibia by 2 crossed screws, and a long leg cast is used for 2 months (7).

Disadvantages of patella bone block include the possibility of patella fracture and recurrence of previous knee hyperextension due to the failure of bone block (7). It also disturbs the normal anatomy of the knee joint, making future reconstructions difficult. Two months of wearing a long leg cast, as recommended by the authors in bone block surgery (7), is another drawback of this technique, as it may further deteriorate the already weak muscles.

Supracondylar femoral flexion osteotomy is another method of treatment for genu recurvatum, which is commonly performed in primary poliomyelitis in the adolescent population with good results (17). However, to the best of our knowledge, this method has not been used in PPS in adult populations. In this technique, an open-wedge osteotomy is made in the supracondylar area of the femur, and an allograft is used to fill the resulting gap. Also, a plate is used to secure the osteotomy site at the end of surgery.

One of the advantages of supracondylar femoral flexion osteotomy is that there is no need for a long leg cast after surgery and rehabilitation can start very soon. This is very important considering the neuromuscular problems of these patients. In fact, use of a long leg cast in the healing process is associated with the severe loss of power in the already weak muscles. In addition, osteotomy restores the normal anatomy of the knee joint, preserves the bone stock, and relives stretching of the posterior soft tissue structures. This technique becomes even more valuable when the patient has a history of supracondylar extension osteotomy for the correction of flexion contracture, as in the present case.

\section{Footnote}

Authors' Contributions: All authors were involved in diagnosis, treatment, manuscript draft preparing and its revision.

\section{References}

1. Jubelt B. Post-Polio Syndrome. Curr Treat Options Neurol. 2004;6(2):8793. [PubMed: 14759341].

2. Takemura J, Saeki S, Hachisuka K, Aritome K. Prevalence of post-polio syndrome based on a cross-sectional survey in Kitakyushu, Japan.JRehabil Med. 2004;36(1):1-3. [PubMed:15074431].

3. Sunnerhagen KS, Grimby G. Muscular effects in late polio. Acta Physiol Scand. 2001;171(3):335-40. doi: 10.1046/j.1365-201x.2001.00836.x. [PubMed: 11412146].

4. Gonzalez H, Khademi M, Andersson M, Wallstrom E, Borg K, Olsson T. Prior poliomyelitis-evidence of cytokine production in the central nervous system. J Neurol Sci. 2002;205(1):9-13. [PubMed: 12409177].

5. Ivanyi B, Nollet F, Redekop WK, de Haan R, Wohlgemuht M, van Wijngaarden JK, et al. Late onset polio sequelae: Disabilities and handicaps in a population-based cohort of the 1956 poliomyelitis outbreak in The Netherlands. Arch Phys Med Rehabil. 1999;80(6):687-90. doi: 10.1016/s0003-9993(99)90173-9.

6. Mehta SN, Mukherjee AK. Flexion osteotomy of the femur for genu recurvatum after poliomyelitis. Bone Joint J. 1991;73(2):200-2.

7. Men HX, Bian CH, Yang CD, Zhang ZL, Wu CC, Pang BY. Surgical treatment of the flail knee after poliomyelitis. Bone Joint J. 1991;73(2):195-9.

8. Van Allen MW. Aids to the examination of the peripheral nervous system. Arch Neurol. 1977;34(1):61. 
9. Wiechers DO, Hubbell SL. Late changes in the motor unit after acute poliomyelitis. Muscle Nerve. 1981;4(6):524-8. doi: 10.1002/mus.880040610. [PubMed: 6273721]

10. Dalakas MC. Pathogenetic mechanisms of post-polio syndrome: morphological, electrophysiological, virological, and immunological correlations. Ann N Y Acad Sci. 1995;753:167-85. [PubMed: 7611626].

11. Inaba M. Control dysfunction. 3. Bracing the unstable knee and ankle in hemiplegia. Phys Ther. 1967;47(9):838-43. [PubMed: 6051082].

12. Perry J. Lower-extremity bracing in hemiplegia. Clin Orthop Relat Res. 1969;63:32-8. [PubMed: 5781120].

13. Gill AB. Operation for correction of paralytic genu recurvatum. JBJS.
1931;13(1):49-53.

14. Campbell WC, Mitchell JI. Operative Treatment of Paralytic Genu Recurvatum. Ann Surg. 1932;96(6):1055-64. [PubMed: 17866893].

15. Mayer L. An operation for the cure of paralytic genu recurvatum. $J$ Bone Joint Surg Am. 1930;12(4):845-52.

16. Campbell WC. An Operation for the Correction and Prevention of Paralytic Genu Recurvatum. J Am Med Assoc. 1918;71(12):967. doi: 10.1001/jama.1918.26020380003002b.

17. Herring JA. Tachdjian's pediatric orthopaedics: from the Texas Scottish Rite Hospital for children. Elsevier Health Sciences; 2013. 